\title{
Influencia de los parámetros de corte en el desgaste del flanco de insertos recubiertos durante el torneado del acero AISI 316L
}

\section{(Influence of the cutting parameters on flank wear of coated inserts during turning of AISI 316L)}

\author{
Yusimit Zamora Hernández ${ }^{1}$, Yoandrys Morales Tamayo ${ }^{1}$, Arlys Michel Lastre Aleaga ${ }^{2}$, \\ Roberto Beltrán Reyna ${ }^{3}$
}

\begin{abstract}
Resumen:
La mejora continua de los procesos de fabricación es fundamental para alcanzar niveles óptimos de productividad, calidad y corte en la producción de componentes y productos. Esta investigación tiene como objetivo determinar la progresión del desgaste del flanco de la herramienta de corte durante el torneado en seco de alta velocidad para piezas de acero AISI 316L. Los datos experimentales fueron adquiridos utilizando dos niveles de avance de corte, dos niveles de material, tres de velocidad de corte y cuatro de tiempos principales de corte. Un microscópio electrónico de barrido (SEM) fue utilizado para medir y analizar el desgaste de las herramientas de corte. Los resultados fueron comparados utilizando el análisis de varianza y el análisis de regresión múltiple para describir la relación entre las variables utilizadas en el estudio. En el análisis se demostró que el inserto de tres capas no sobrepasó el criterio de fin de vida del desgaste, mientras que el inserto de una capa sufrió un desgaste elevado para la mayor velocidad de corte. Se encontró que existe una relación entre los datos experimentales y los valores predichos para el desgaste del flanco con un error promedio general de 4,1182 \%.
\end{abstract}

Palabras clave: Torneado de alta velocidad; Desgaste de flanco; Acero inoxidable AISI 316L.

\begin{abstract}
:
The continuous improvement of manufacturing processes is critical to achieve optimum levels of productivity, quality and cut production of components and products. This research aims to determine the cutting tool flank wearing progression, during a high speed dry turning, for AISI $316 \mathrm{~L}$ steel parts. Experimental data were acquired using two cutting feed levels, two material levels, three cutting speeds, and four principal cutting times. A scanning electron microscope (SEM) was used to measure and analyze the wear of the cutting tools. Results were compared using analysis of variance and multiple regression for describing the relation between the variables used in the study. The analysis showed that the three layers coating insert did not exceed the end of life wearing criterion, while the one layer insert suffered a catastrophic wearing at the highest cutting speed. It was found that a relation exists between the experimental data and the predicted values for flank wear with a general average error of $4.1182 \%$.
\end{abstract}

Keywords: High speed turning; Flank wear; AISI 316L.

\footnotetext{
${ }^{1}$ Universidad de Granma, Ingeniería Mecánica, Bayamo - Cuba. (yzamorah@udg.co.cu)

${ }^{2}$ Universidad Tecnológica Equinoccial- Sede Santo Domingo, Ciencias de la Ingeniería, Santo Domingo de los Tsachilas- Ecuador. (arlys.lastre@ute.edu.ec)

${ }^{3}$ Universidad de las Fuerzas Armadas, ESPE Extensión Latacunga-Ecuador.(rfbeltrnr@espe.edu.ec)
} 


\section{Introducción}

La demanda de componentes mecánicos de alta calidad, gran exactitud y menores tiempos de entrega para sistemas de elevado desempeño ha aumentado considerablemente en los últimos años a nivel mundial. El avance alcanzado en las máquinas herramienta, en las herramientas de corte y en las tecnologías de maquinado han posibilitado la utilización del maquinado de alta velocidad (HSM).Este posibilita una mayor velocidad de remoción de material, reduce los tiempos de maquinado, el número de máquinas herramientas y garantiza un elevado acabado superficial, disminuyendo las fuerzas de corte y la vida útil de la herramienta de corte(Hernández et al., 2011).

La importancia de evaluar el desgaste y predecir la vida de la herramienta radica en los efectos indeseables que provoca como: una menor exactitud dimensional de la pieza terminada, tensiones residuales superficiales, deficiente rugosidad superficial y aumento de las vibraciones durante el proceso de corte(Suresh et al., 2012b).

Muchas soluciones para aumentar la vida de la herramienta han estado dirigidas a disminuir la temperatura en la interface herramienta-pieza mediante la utilización y desarrollo de fluidos de corte de diferentes naturalezas. La utilización de estos encarece los costos de fabricación, provoca daños en la salud de los operarios y afecta el medio ambiente. Las nuevas tecnologías para atenuar los efectos perjudiciales de los fluidos de corte se sustentan en el corte en seco, en la mínima lubricación y en el desarrollo de nuevas fórmulas de fluidos (Cordes, 2012).

En los últimos años las investigaciones relacionadas con la evaluación de la vida útil de la herramienta han estado dirigidas a efectuar el corte de metales refrigerado, sin embargo son escasas las investigaciones relacionadas con el mecanizado en seco a altas velocidades utilizadas en el torneado de aceros inoxidables austeníticos. A continuación se muestra un resumen de los trabajos investigativos más relevantes en la elaboración de los aceros inoxidables austeníticos.

Korkutet al.(2004), investigaron la influencia de la velocidad de corte en el desgaste de la herramienta y en la rugosidad superficial durante la elaboración en seco del acero inoxidable AISI 304 con herramientas multicapas ( $\mathrm{TiC}, \mathrm{TiCN}, \mathrm{Al} 2 \mathrm{O} 3, \mathrm{TiN}$ ) utilizando velocidades de corte entre 120 y $180 \mathrm{~m} / \mathrm{min}$. Por otro lado, Ciftci en el(2006),evaluó la rugosidad superficial y las fuerzas de corte para herramientas revestidas durante el mecanizado en seco de los aceros inoxidables AISI $316 \mathrm{~L}$ y AISI 304 empleando velocidades de corte entre 120 y $210 \mathrm{~m} / \mathrm{min}$. Estos autores utilizaron velocidades de corte bajas en sus análisis.

Lin en el(2008), evaluó el comportamiento de la rugosidad superficial para aplicar el torneado de acabado en seco de los aceros AISI 303 y 304 utilizando velocidades de corte entre 250 y 450 $\mathrm{m} / \mathrm{min}$. Este autor utiliza elevadas velocidades de corte pero no evaluó el desgaste de la herramienta. 
En su artículo Xavior y Adithan en el(2009), determinaron la influencia de los fluidos de corte en el desgaste de la herramienta y en la rugosidad superficial durante el torneado de AISI 304 con herramientas de carburos y velocidades de corte de 38,95; 61,35 y 97,38 m/min. Estos autores analizaron bajas velocidades. Galanis y Manolakos en el (2010)desarrollaron un modelo para predecir la rugosidad superficial en cabezas femorales en el torneado del acero inoxidable AISI $316 \mathrm{~L}$ utilizando velocidades hasta $440 \mathrm{~m} / \mathrm{min}$. Estos autores, aunque trabajaron en la gama de la alta velocidad no midieron el desgaste de la herramienta de corte.

Mahdavinejad y Saeedy en el (2011) realizaron un estudio experimental para optimizar los parámetros de corte del torneado en seco y con fluidos de corte del acero inoxidable AISI 304. Este estudio utilizaron herramientas de carburo de tungsteno con velocidades de corte entre $100 \mathrm{y}$ $200 \mathrm{~m} / \mathrm{min}$.

Leppert y Peng en el(2012) analizaron la tensión residual producida en la capa superficial producida durante el torneado del acero inoxidable AISI 316 con herramientas recubiertas. En el maquinado emplearon velocidades de corte hasta $255 \mathrm{~m} / \mathrm{min}$. Estos autores no incluyeron en su investigación altas velocidades de corte, ni midieron el desgaste de los insertos.

Naves y otros investigadores analizaron la aplicación de fluidos de corte a alta presión en las herramientas de corte con el objetivo de alargar la vida de estas. El factor analizado fue el desgaste del flanco para $300 \mathrm{~m} / \mathrm{min}$ como velocidad de corte(Naves et al., 2013). En el 2014 Gerth y otros, realizan una investigación para profundizar en el estudio del proceso de adhesión que se produce entre la viruta y la arista de corte de la herramienta. La operación corresponde al corte ortogonal y la velocidad de corte que utiliza es de $150 \mathrm{~m} / \mathrm{min}$ (Gerth et al., 2014). Estas dos últimas investigaciones no estudian el rango de alta velocidad y además utilizan los fluidos de corte para alargar la vida útil de las herramientas.

Del análisis anterior se aprecian que las investigaciones se centran en el estudio de la rugosidad superficial, las fuerzas de corte y los menos estudiados son las vibraciones mecánicas, las deformaciones superficiales y el desgate de la herramienta en el mecanizado de alta velocidad en seco de los aceros inoxidables.

Por tanto, esta investigación tiene como objetivo investigar la evolución del desgaste del flanco respecto al tiempo de maquinado de dos insertos de carburo recubierto durante el torneado en seco a altas velocidades del acero inoxidable AISI 316L.

\section{Materiales y Métodos}

El torneado experimental fue ejecutado en condiciones secas, con el uso del torno multifuncional tipo Okuma Multus B200-W con una potencia del motor de 15 kW. La rotación del husillo oscila entre 50 y 5000 rpm y el peso máximo que soporta sobre el cabezal fijo es de $110 \mathrm{~kg}$. 
La probeta seleccionada fue de acero inoxidable AISI 316L muy utilizado en la fabricación de productos resistentes a la corrosión y resistentes a altas temperaturas(Gaitonde et al., 2008). La composición química se puede observar es la tabla 1.

Tabla 1. Composición química del acero AISI 316L.

\begin{tabular}{|c|c|c|c|c|c|c|c|c|c|}
\hline Elemento & C & $\mathrm{Si}$ & $\overline{M n}$ & $\mathrm{Cr}$ & Mo & $\mathrm{Ni}$ & $P$ & S & $\mathrm{N}$ \\
\hline $\begin{array}{c}\text { Composición } \\
(\%)\end{array}$ & $\frac{10}{0}$ & 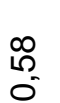 & 욛 & 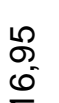 & $\begin{array}{l}\text { L } \\
\text { N }\end{array}$ & $\begin{array}{l}\infty \\
0 \\
0 \\
0\end{array}$ & $\bar{g}$ & స్తి & 员 \\
\hline
\end{tabular}

Las probetas de $100 \mathrm{~mm}$ de diámetro y $200 \mathrm{~mm}$ de longitud fueron torneadas con insertos recubiertos con marcación Sandvik, GC1115 y GC2015. Los recubrimientos de (TiCN- $\left.\mathrm{Al}_{2} \mathrm{O}_{3}-\mathrm{TiN}\right)$ con un espesor de $15 \mu \mathrm{m}$ correspondieron al inserto GC1115 y para el inserto tipo GC2015 su recubrimiento fue de TiN con $5 \mu \mathrm{m}$ de espesor.

La geometría de los insertos fue CCMT 1204 04-MF con rompevirutas, el portaherramienta marca Sandvik de código C6-SCLCL-45065-12 y un adaptador con código C6-391.01-63 060. El ángulo de incidencia principal fue de $7^{\circ}$, el ángulo de ataque fue de $0^{\circ}$ y el radio de la punta fue de 0,4 $\mathrm{mm}$. La dureza de los insertos determinada con un microdurómetro marca SHIMADZU fue 1755 HV GC1115 y 1404 HV para el inserto GC2015.

El análisis factorial completo fue el procedimiento utilizado para determinar la relación entre las variables independientes (parámetros de corte) y la variable dependiente (desgaste del flanco (w)). Un total de 64 ensayos para dos replicas fueron desarrollados con dos niveles de velocidades de corte $(v)$, cuatro niveles de tiempo $(T)$, dos niveles de avances de corte (f) y dos niveles de material de la herramienta, en la tabla 2 aparecen las variables estudiadas.

Tabla 2. Variables consideradas en el estudio.

\begin{tabular}{|c|c|c|c|}
\hline Velocidad de & \multicolumn{2}{|c|}{ Avance $\mathrm{f}(\mathrm{mm} / \mathrm{rev})$} & Tiempo principal de \\
\hline \multirow{4}{*}{400} & \multirow{4}{*}{0,08} & \multirow{4}{*}{0,16} & 2 \\
\hline & & & 3 \\
\hline & & & 4 \\
\hline & & & 5 \\
\hline \multirow{4}{*}{450} & \multirow{4}{*}{0,08} & \multirow{4}{*}{0,16} & 0,6 \\
\hline & & & 1,2 \\
\hline & & & 2 \\
\hline & & & 3 \\
\hline
\end{tabular}


En esta investigación fue asumido como criterio límite de desgaste del flanco 0,2 mm teniendo en cuenta los resultados de la revisión de la literatura científica además de que se trata de una elaboración de acabado en seco y a alta velocidad de corte(Cordes, 2012).

Se realizaron pruebas iniciales para determinar el estado de los instrumentos de medición, máquinas y del personal encargado de la experimentación. Se cilindró la pieza comenzando con un filo nuevo y el corte fue interrumpido en diferentes valores de tiempo, según la Tabla 2, para medir el desgaste del flanco utilizando un microscopio electrónico de barrido marca JEOL. La medición del desgaste fue efectuada acorde a la norma ANSI/ASME B94.55M, además se utilizaron varias probetas de acero inoxidable AISI 316L para evitar cualquier efecto de confusión entre las mismas y la selección de la pieza para cada ensayo fue completamente aleatoria.

\section{Discusión de los Resultados}

El objetivo del trabajo es evaluar la evolución del desgaste del flanco respecto al tiempo principal de maquinado de dos insertos de carburo recubierto para dos velocidades y dos avances de corte.

En las figuras 1 y 2 se muestran las mediciones del desgaste del flanco de los insertos para 400 $\mathrm{m} / \mathrm{min}$ y $450 \mathrm{~m} / \mathrm{min}$ de velocidad de corte, para 5 y $3 \mathrm{~min}$ de tiempo de maquinado respectivamente. Se observa que para $v=400 \mathrm{~m} / \mathrm{min}$ y $f=0,16 \mathrm{~mm} / \mathrm{rev}$ los insertos GC1115 y GC2015 no exceden el criterio de fin de vida del desgaste del flanco (200 $\mu \mathrm{m})$. Para la velocidad de $450 \mathrm{~m} / \mathrm{min}$ y avance $0,16 \mathrm{~m} / \mathrm{min}$ el inserto GC1115 sufre un gran desgaste sobrepasando por mucho el criterio de fin de vida del desgaste del flanco y el inserto GC2015 se aproxima a este límite, aunque no lo sobrepasa.

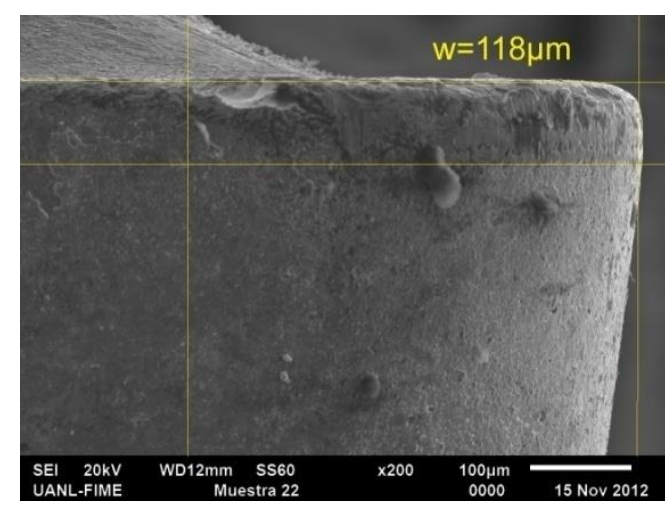

(a)

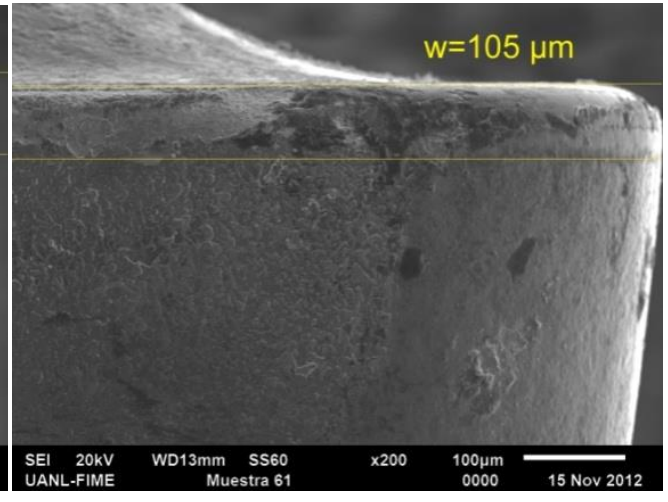

(b)

Figura 1. Desgaste del flanco $(w)$ de los insertos para $f=0,16 \mathrm{~mm} / \mathrm{rev}, T=5 \mathrm{~min}$ y $\mathrm{v}=400$ $\mathrm{m} / \mathrm{min}$ donde: (a) GC1115 y (b) GC2015. 


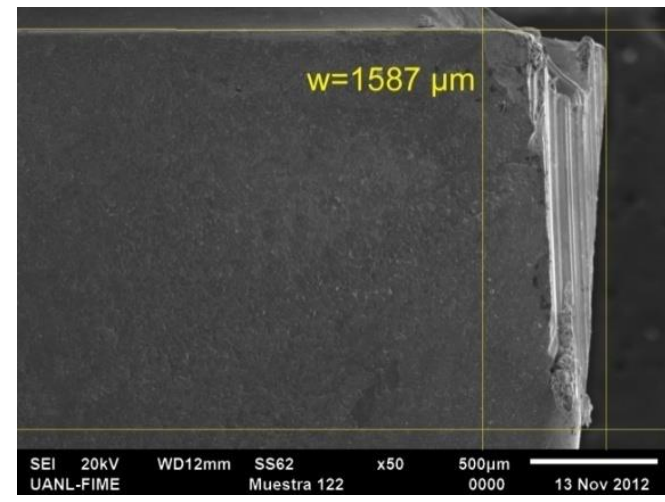

(a)

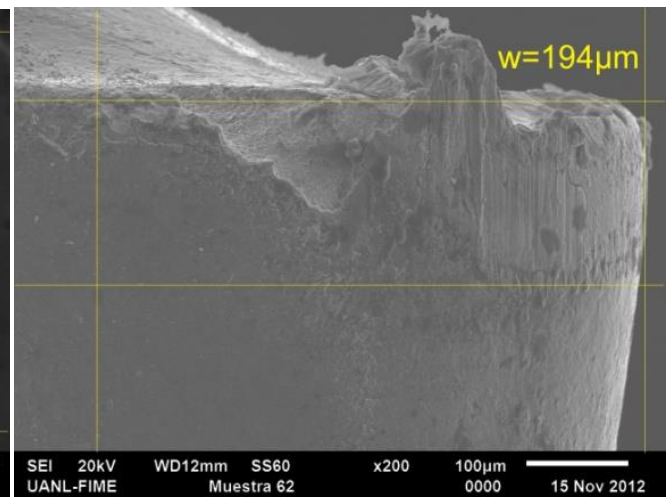

(b)

Figura 2. Desgaste del flanco $(\mathrm{w})$ de los insertos para $f=0,16 \mathrm{~mm} / \mathrm{rev}, \mathrm{T}=3 \mathrm{~min}$ y $\mathrm{v}=450$ $\mathrm{m} / \mathrm{min}$ donde: (a) GC1115 y (b) GC2015.

La utilización de elevadas velocidades de corte provoca un aumento de la temperatura en la herramienta de corte, como consecuencia debilita la arista de corte(Bartarya \& Choudhury, 2012).Los insertos maquinados a $400 \mathrm{~m} / \mathrm{min}$ mostraron desgaste por abrasión, adhesión y mueca. Para el caso de la velocidad de $450 \mathrm{~m} / \mathrm{min}$, el inserto GC1115 presenta un excesivo desgaste revelando abrasión, adhesión, difusión, deformación plástica, y fractura del borde cortante, mientras que el GC2015 muestra abrasión, adhesión, difusión, y mueca, conclusión similar a la obtenida por Jianxin et al(2011).

En la figura 3a se muestra la evolución del desgaste del flanco de los insertos para $v=400 \mathrm{~m} / \mathrm{min}$, se aprecia un mejor comportamiento del inserto GC1115 hasta 4 min de corte. Para los 5 min de maquinado el inserto GC2015 obtuvo el mejor resultado en cuanto al desgaste, ninguno de los dos insertos alcanzó el límite del criterio de fin de vida. Para la velocidad de corte de $450 \mathrm{~m} / \mathrm{min}$ (figura 3b) el mejor desempeño hasta 1,2 min de corte fue también para el inserto GC1115, en cambio para los tiempos restantes el desgaste del flanco en sus filos de corte fue superior hasta llegar a desmoronarse antes de alcanzar el tiempo final de maquinado. Para el caso del avance en la figura $4 \mathrm{a}$ y $4 \mathrm{~b}$ es apreciable que el comportamiento del desgaste del flanco fue favorable cuando se utilizó el avance de $0,08 \mathrm{~mm} / \mathrm{rev}$ en las dos velocidades.

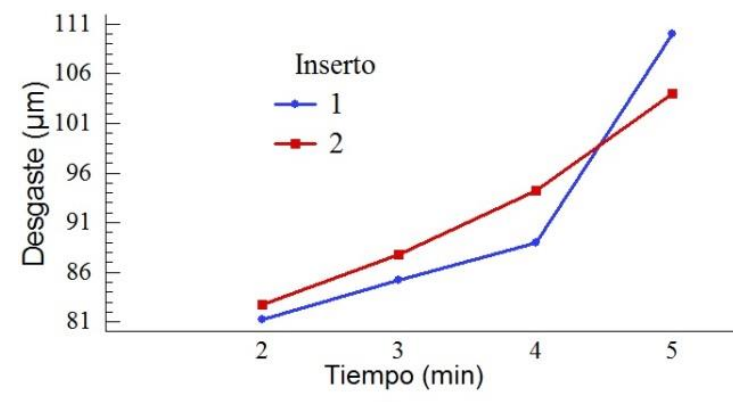

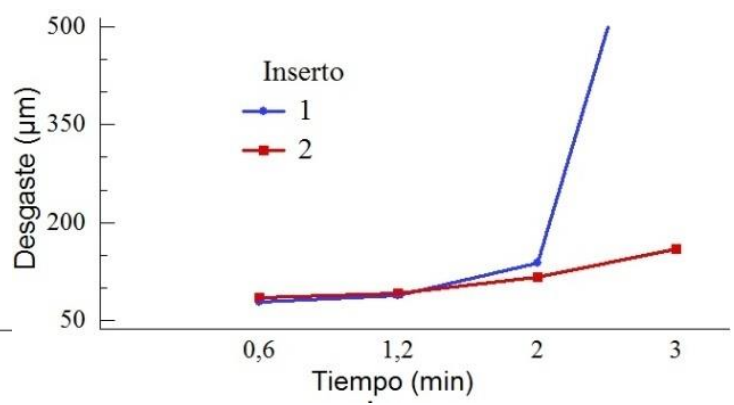

b

Figura 3. Evolución del desgaste del flanco de los insertos GC1115 (1) y GC2015 (2) para v = $400 \mathrm{~m} / \mathrm{min}$ (a) y $v=450 \mathrm{~m} / \mathrm{min}$ (b). 


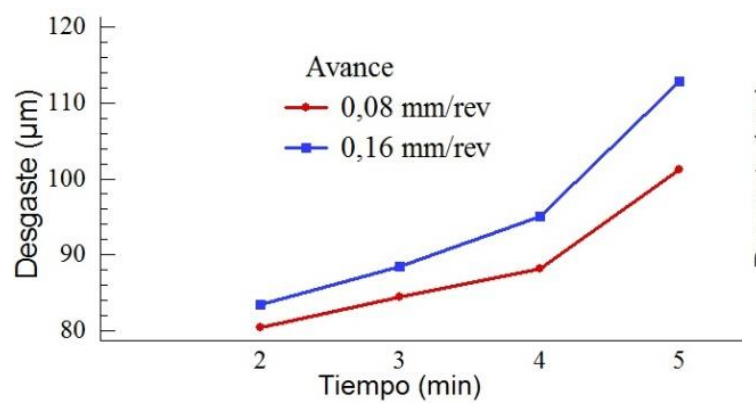

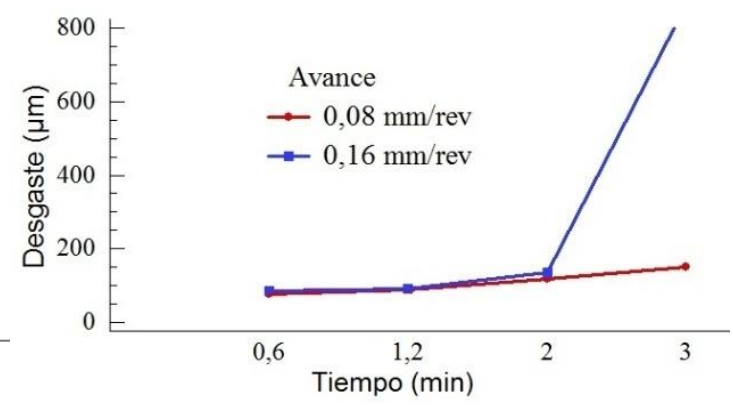

b

Figura 4. Evolución del desgaste del flanco con la utilización de los avances para $v=400$ $\mathrm{m} / \mathrm{min}$ (a) y $v=450 \mathrm{~m} / \mathrm{min}$ (b).

Se observó que la herramienta de menor desgaste GC2015 es coincidentemente la de menor dureza, debido a que posee tres recubrimientos específicos para mejorar la mecánica del corte. La capa compuesta por $\mathrm{Ti}(\mathrm{N}, \mathrm{C})$ le proporciona resistencia al desgaste y estabilidad térmica, la capa de $\mathrm{Al}_{2} \mathrm{O}_{3}$ le suministra resistencia al calor y al desgaste cráter y el recubrimiento de TiN le aporta resistencia al calor y un bajo coeficiente de fricción(Suresh et al., 2012a). Además la herramienta de mayor dureza solo presenta un recubrimiento de $5 \mu \mathrm{m}$ de espesor mientras que la suma de los recubrimientos del inserto de tres capas alcanza $15 \mu \mathrm{m}$ de espesor.

Se realizó el análisis de varianza para determinar los factores y las interacciones estadísticamente significativas en el desgaste del flanco y además se realizó un análisis de regresión utilizando el software STATGRAPHICS Plus versión 5.1

\section{Análisis de varianza factorial y de regresión.}

El análisis del estudio experimental realizado para la velocidad de corte de $400 \mathrm{~m} / \mathrm{min}$ se presenta en la tabla 3. Se midió la contribución de cada factor eliminando los efectos del resto de los factores. La importancia significativa de las variables para un $95 \%$ de confianza se comprueba determinando los p-valores inferiores a 0,05. Resultaron significativos el avance de corte, el tiempo de maquinado y la interacción entre estas dos variables, por el contrario los tipos de insertos no resultaron tener un efecto significativo en consecuencia que su $p$-valor es superior a 0,05 .

Tabla 3. Análisis de varianza factorial de desgaste del flanco de los insertos para $400 \mathrm{~m} / \mathrm{min}$.

\begin{tabular}{|l|c|c|c|c|c|}
\hline \multicolumn{1}{|c|}{ Fuente } & $\begin{array}{c}\text { Suma de } \\
\text { cuadrados }\end{array}$ & $\begin{array}{c}\text { Grado de } \\
\text { libertad }\end{array}$ & $\begin{array}{c}\text { Cuadrado } \\
\text { medio }\end{array}$ & Cociente-F & p-valor \\
\hline Avance de corte & 300,12 & 1 & 300,125 & 59,25 & 0,0000 \\
\hline Inserto & 3,12 & 1 & 3,125 & 0,62 & 0,4407 \\
\hline Tiempo de maquinado & 2503,75 & 3 & 834,583 & 165,61 & 0,0000 \\
\hline Inserto-Tiempo & 221,62 & 3 & 73,875 & 14,66 & 0,0000 \\
\hline Residuo & 95,75 & 19 & 5,039 & & \\
\hline Total (corregido) & 3202,00 & 31 & & & \\
\hline
\end{tabular}


Se realizó un análisis de regresión para describir la relación entre el desgaste del flanco de cada inserto, el tiempo de maquinado y el avance de corte, para obtener la ecuación del modelo ajustado. Además se determinó el coeficiente de determinación ajustado $\left(R^{2}\right)$ que representa la bondad del ajuste. En la figura 5 y 6 se muestran las ecuaciones de regresión de los modelos ajustados para cada inserto y el coeficiente de determinación para $400 \mathrm{~m} / \mathrm{min}$ de velocidad de corte a través de una superficie respuesta. Además se presenta el tiempo de vida útil de cada inserto (Tvu) para cada avance de corte teniendo en cuenta el criterio de fin de vida (200 $\mu \mathrm{m})$.

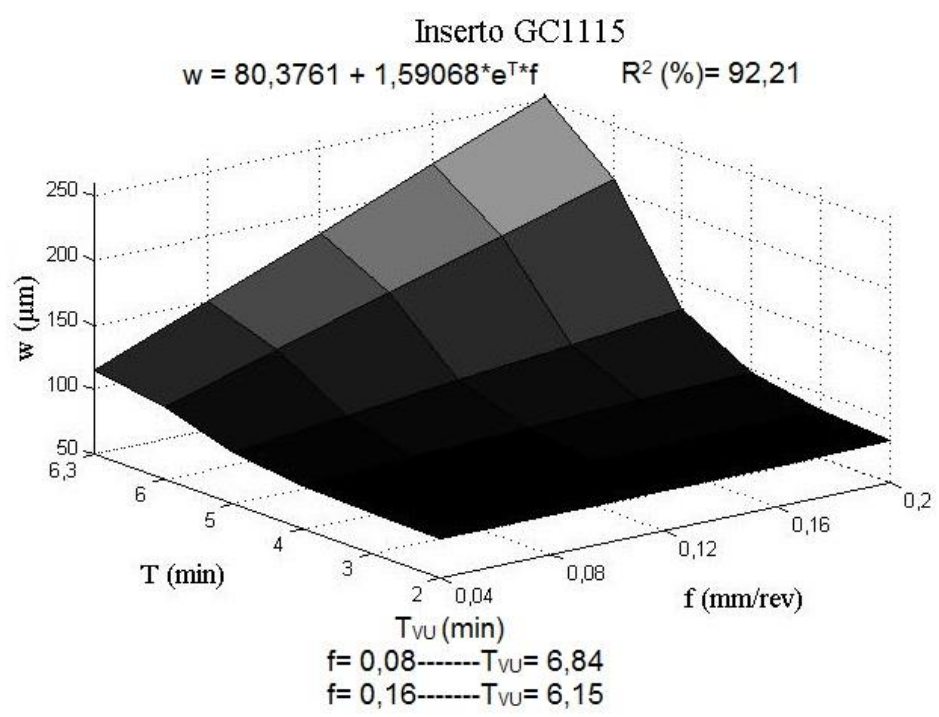

Figura 5. Análisis del desgaste del flanco del inserto GC1115 para v $=400 \mathrm{~m} / \mathrm{min}$.

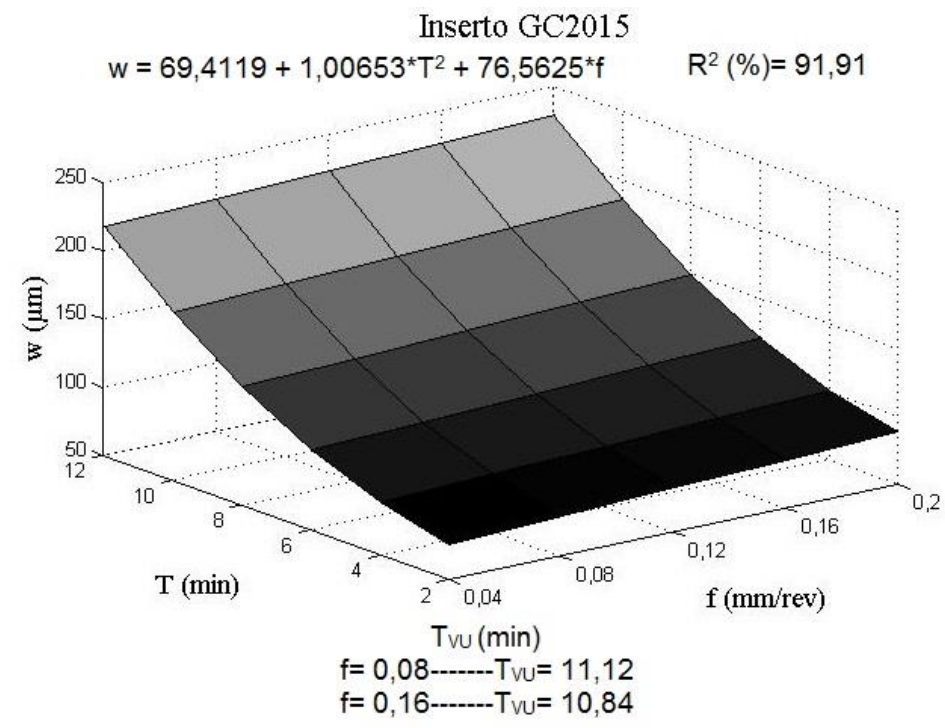

Figura 6. Análisis del desgaste del flanco del inserto GC2015 para v $=400 \mathrm{~m} / \mathrm{min}$.

La comparación en el análisis de la varianza (tabla 4) del desgaste del flanco para $\mathrm{v}=450 \mathrm{~m} / \mathrm{min}$ muestra que los insertos, el tiempo de maquinado y la interacción entre ellos tienen un efecto estadísticamente significativo en el desgaste del flanco para un nivel de confianza del 95,0\%, sin embargo el avance de corte no tuvo un efecto significativo para este nivel de confianza. Los 
resultados del análisis de regresión para describir la relación entre el desgaste del flanco de los insertos, el avance de corte y el tiempo de maquinado para velocidad de corte $(450 \mathrm{~m} / \mathrm{min})$ se observan en la superficie respuesta que se muestra en las figuras 7 y 8 . Además se puede observar el tiempo de vida útil de cada inserto (Tvu) para cada avance de corte utilizado.

Tabla 4. Análisis de varianza factorial de desgaste del flanco de los insertos para $450 \mathrm{~m} / \mathrm{min}$.

\begin{tabular}{|l|c|c|c|c|c|}
\hline \multicolumn{1}{|c|}{ Fuente } & $\begin{array}{c}\text { Suma de } \\
\text { cuadrados }\end{array}$ & $\begin{array}{c}\text { Grado de } \\
\text { libertad }\end{array}$ & $\begin{array}{c}\text { Cuadrado } \\
\text { medio }\end{array}$ & Cociente-F & p-valor \\
\hline Avance de corte & 275097,0 & 1 & 275097,0 & 7,94 & 0,1100 \\
\hline Inserto & 243428,0 & 1 & 243428,0 & 7,02 & 0,0158 \\
\hline Tiempo de maquinado & 97439,0 & 3 & 324780,0 & 9,37 & 0,0005 \\
\hline Inserto-Tiempo & 705615,0 & 3 & 235205,0 & 6,79 & 0,0027 \\
\hline Residuo & 658405 & 19 & 34652,9 & & \\
\hline Total (corregido) & 3,616 & 31 & & & \\
\hline
\end{tabular}

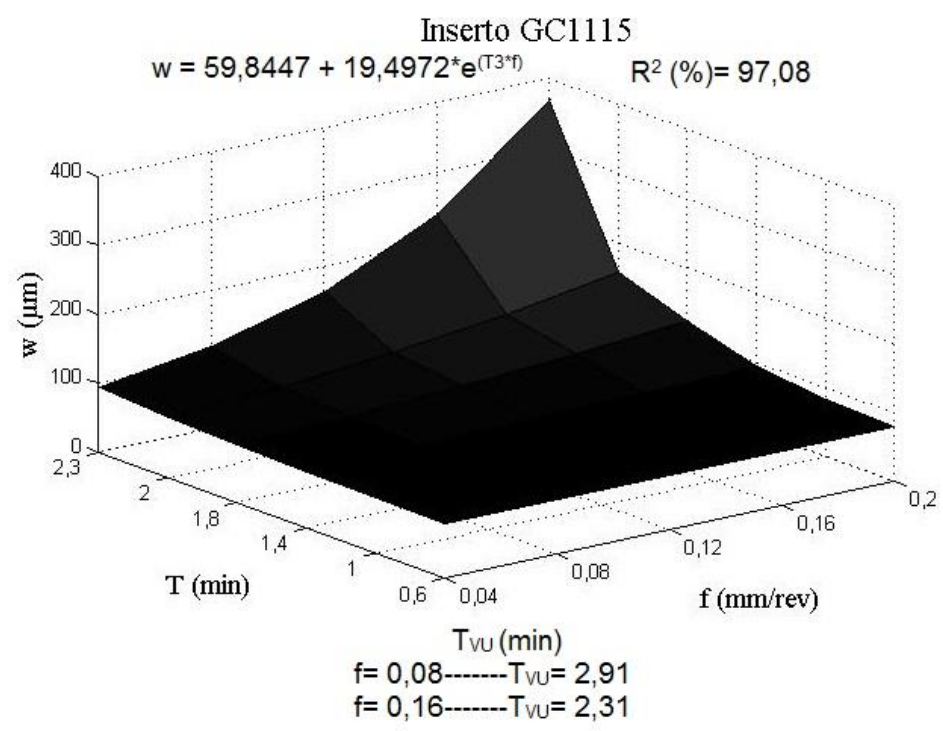

Figura 7. Análisis del desgaste del flanco del inserto GC1115 para $v=450 \mathrm{~m} / \mathrm{min}$. Inserto $\mathrm{GC} 2015$

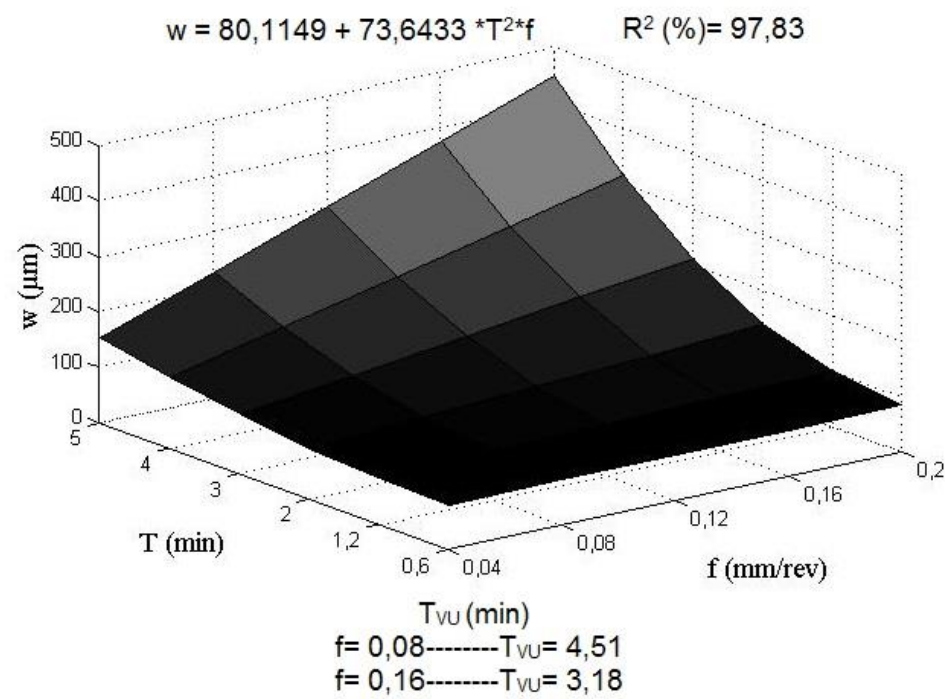

Figura 8. Análisis del desgaste del flanco del inserto GC2015 para v = $450 \mathrm{~m} / \mathrm{min}$. 


\section{Análisis de los errores de los modelos propuestos en la investigación}

La exactitud y precisión de un modelo es evaluada y comprobada comúnmente por la ecuación 1 (Jiao et al., 2004; Asiltürk \& Çunka, 2011; Suresh et al., 2012a).

$\mathrm{E}_{\text {medio }}=\left(\frac{1}{\mathrm{~N}} \sum_{\mathrm{i}}^{\mathrm{N}}\left|\frac{\mathrm{t}_{\mathrm{i}}-\mathrm{t}_{0}}{\mathrm{t}_{\mathrm{o}}}\right| \times 100\right)$

Donde: $\mathrm{E}_{\text {medio }}$, error medio absoluto.

$t_{i}$, son los valores reales obtenidos por experimentación.

$t_{0}$, son los valores predichos por el modelo evaluado en cuestión.

Los errores medios absolutos de los modelos obtenidos se muestran en la tabla 5 . En ella se puede apreciar que los modelos alcanzaron errores medios absolutos menores al $15 \%$.

Tabla 5. Errores medios absolutos (\%) obtenidos por regresión múltiple.

\begin{tabular}{|c|c|c|}
\hline Velocidad & Inserto & $\mathrm{E}_{\text {medio }}(\%)$ \\
\hline \multirow{2}{*}{400} & GC1115 & 1,662 \\
\cline { 2 - 3 } & GC2015 & 1,182 \\
\hline \multirow{2}{*}{450} & GC1115 & 11,979 \\
\cline { 2 - 3 } & GC2015 & 1,650 \\
\hline \multicolumn{2}{|c|}{ Promedio General } & 4,1182 \\
\hline
\end{tabular}

\section{Conclusiones y Recomendaciones}

En este trabajo los datos experimentales fueron adquiridos utilizando dos niveles de avance de corte, dos niveles de material, tres de velocidad de corte y cuatro de tiempo. Basados en los análisis y resultados del estudio del desgaste de la herramienta durante el torneado del acero AISI 316L se llegó a las siguientes conclusiones:

- El desgaste del flanco aumentó con el tiempo de maquinado para las velocidades de corte utilizadas en el estudio.

- Para las velocidades de corte estudiadas, el mejor desempeño corresponde al inserto GC2015 con tiempos de vida útil superiores al inserto GC1115.

- El desgaste del flanco tuvo mejor comportamiento para el avance de 0,08 mm/rev en todas las velocidades empleadas en este estudio.

- El análisis de varianza factorial demostró que el avance de corte y el tiempo de maquinado tienen un efecto significativo para un nivel de confianza del 95\%.

- Se encontró que existe una relación entre los datos experimentales y los valores predichos para el desgaste del flanco con un error promedio general de $4,1182 \%$. 
- Teniendo en cuenta los resultados de los errores y los coeficientes de determinación se puede expresar que los modelos obtenidos por regresión son adecuados para utilizarse como estimados del desgaste del flanco cuando se utilizan los parámetros de corte.

\section{Agradecimientos}

Los autores agradecen a la SEP por proporcionar la beca postgraduada de investigación en la Universidad Autónoma de Nuevo León (UANL) en México. Se le agradece al Centro de Investigación e Innovación en Ingeniería Aeronáutica por el apoyo financiero, tecnológico y por todas las facilidades brindadas para el desarrollo del trabajo de investigación. Se agradece al Departamento de Ciencias Técnicas de la Universidad de Granma por el apoyo brindado.

\section{Bibliografía}

Asiltürk, I., \& Çunka, M. (2011). Modeling and prediction of surface roughness in turning operations using artificial neural network and multiple regression method. Expert Systems with Applications, 38, 5826-5832. doi: 10.1016/j.eswa.2010.11.041

Bartarya, G., \& Choudhury, S. K. (2012). State of the art in hard turning. International Journal of Machine Tools and Manufacture, 53(1), 1-14. doi: 10.1016/j.jmachtools.2011.08.019

Ciftci, I. (2006). Machining of austenitic stainless steels using CVD multi-layer coated cemented carbide tools. Tribology International, 39(6), 565-569. doi: 10.1016/j.triboint.2005.05.005

Cordes, S. E. (2012). Thermal stability of V-alumina PVD coatings and analysis of their performance in machining of austenitic stainless steels. CIRP Journal of Manufacturing Science and Technology, 5(1), 20-25. doi: 10.1016/j.cirpj.2011.11.003

Gaitonde, V., Karnik, S., Siddeswarappa, B., \& Achyutha, B. (2008). Integrating Box-Behnken design with genetic algorithm to determine the optimal parametric combination for minimizing burr size in drilling of AISI 316L stainless steel. The International Journal of Advanced Manufacturing Technology, 37(3), 230-240. doi: 10.1007/s00170-007-0957-4

Galanis, N., \& Manolakos, D. (2010). Surface roughness prediction in turning of femoral head. The International Journal of Advanced Manufacturing Technology, 51(1), 79-86. doi: 10.1007/s00170-010-2616-4

Gerth, J., Gustavsson, F., Collin, M., Andersson, G., Nordh, L. G., Heinrichs, J., et al. (2014). Adhesion phenomena in the secondary shear zone in turning of austenitic stainless steel and carbon steel. Journal of Materials Processing Technology, 214(8), 1467-1481. doi: 10.1016/j.jmatprotec.2014.01.017

Hernández, L. W., Pérez, R., Zambrano, P., Guerrero, M., \& Dumitrescu, P. (2011). Estudio del desgaste del flanco de carburos recubiertos y cermet durante el torneado de alta velocidad en seco del acero AISI 1045. Revista de Metalurgia, 47(3), 262-272. doi: 10.3989/revmetalm.1039 
Jianxin, D., Jiantou, Z., Hui, Z., \& Pei, Y. (2011). Wear mechanisms of cemented carbide tools in dry cutting of precipitation hardening semi-austenitic stainless steels. Wear, 270(7-8), 520527. doi: 10.1016/j.wear.2011.01.006

Jiao, Y., Lei, S., Pei, Z. J., \& Lee, E. S. (2004). Fuzzy adaptive networks in machining process modeling: surface roughness prediction for turning operations. International Journal of Machine Tools \& Manufacture, 44, 1643-1651. doi: 10.1016/j.jmachtools.2004.06.004

Korkut, I., Kasap, M., Ciftci, I., \& Seker, U. (2004). Determination of optimum cutting parameters during machining of AISI 304 austenitic stainless steel. Materials \& Design, 25(4), 303-305. doi: 10.1016/j.matdes.2003.10.011

Leppert, T., \& Peng, R. (2012). Residual stresses in surface layer after dry and MQL turning of AISI 316L steel. Production Engineering, 6(4), 367-374. doi: 10.1007/s11740-012-0389-3

Lin, H. M. (2008). The study of high speed fine turning of austenitic stainless steel. Journal of Achievements in Materials and Manufacturing Engineering, 27(2), 191-194.

Mahdavinejad, R., \& Saeedy, S. (2011). Investigation of the influential parameters of machining of AISI 304 stainless steel. Sadhana, 36(6), 963-970. doi: 10.1007/s12046-011-0055-z

Naves, V. T. G., Da Silva, M. B., \& Da Silva, F. J. (2013). Evaluation of the effect of application of cutting fluid at high pressure on tool wear during turning operation of AISI 316 austenitic stainless steel. Wear, 302(1-2), 1201-1208. doi: 10.1016/j.wear.2013.03.016

Suresh, R., Basavarajappa, S., Gaitonde, V. N., \& Samuel, G. L. (2012a). Machinability investigations on hardened AISI 4340 steel using coated carbide insert. International Journal of Refractory Metals and Hard Materials, 33, 75-86. doi: 10.1016/j.jijmhm.2012.02.019

Suresh, R., Basavarajappa, S., \& Samuel, G. L. (2012b). Some studies on hard turning of AISI 4340 steel using multilayer coated carbide tool. Measurement, 45(7), 1872-1884. doi: 10.1016/j.measurement.2012.03.024

Xavior, M. A., \& Adithan, M. (2009). Determining the influence of cutting fluids on tool wear and surface roughness during turning of AISI 304 austenitic stainless steel. Journal of Materials Processing Technology, 209(2), 900-909. doi: 10.1016/j.jmatprotec.2008.02.068 